\title{
T-odd fragmentation functions
}

\author{
M. Radici ${ }^{\mathrm{a}}$

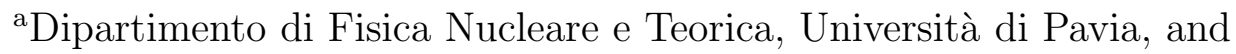 \\ Istituto Nazionale di Fisica Nucleare, Sezione di Pavia, I-27100 Pavia, Italy
}

We discuss the properties of fragmentation functions arising from the interference of two leading hadrons produced inside the same jet in the current fragmentation region of a hard process. For the case of semi-inclusive Deep Inelastic Scattering (DIS), a proper folding of the cross section, integrated over the azimuthal position of the detected hadrons, produces a factorized form that allows for the extraction of the quark transversity distribution at leading twist. Using an extended spectator model, explicit calculations are shown for the hadron pair being two pions with invariant mass inside the $\rho$ resonance width.

Fragmentation functions $(\mathrm{FF})$, like distribution functions (DF), describe the nonperturbative properties of partons when confined inside hadronic bound states at a low scale. Factorization theorems for hard processes, whenever available, ensure their univsersality. Several DF have been experimentally extracted and are parametrized with high accuracy. However, one of them, the quark transversity $h_{1}$, is still unknown because it is connected to soft processes that flip chirality; as such, it is, e.g., unaccessible in inclusive DIS. Among alternative measurements, the single spin asymmetries in semi-inclusive DIS seem more favourable. In fact, a new generation of machines (HERMES, COMPASS, eRHIC) allows for a better resolution in the final state and precise semi-inclusive measurements are becoming feasible. In this context, naive time-reversal odd (for brevity, "T-odd") FF naturally arise because no constraints from time-reversal invariance can be imposed due to the existence of Final State Interactions (FSI) with or inside the residual jet [1]. Some of these T-odd FF are also chiral odd, and they can be shown to represent the natural partner to isolate $h_{1}$ already at leading twist. In a field-theoretical description that assumes factorization, the soft processes linking the fragmenting quark to the observed hadron(s) are defined as matrix elements of nonlocal operators involving quarks and gluons [2]. The simplest one at leading twist is the quark-quark correlator describing the decay of a quark $k$ into a detected hadron $P_{h}$. If the transverse momentum $\vec{P}_{h \perp}$ is measured with respect to the 3-momentum transfer $\vec{q} \| \hat{z}$ and the quark is transversely polarized, a T-odd chiral-odd FF arises and allows for the extraction of $h_{1}$ via a single spin asymmetry (the socalled Collins effect) [3]. However, due to the lack of collinear factorization the soft-gluon radiation needs to be taken into account and can lead to a severe suppression of the effect [4]. Moreover, knowledge of the Collins function implies the difficult task of modelling FSI between the observed hadron and the residual jet [5].

From the theoretical point of view, it is more convenient to select processes where two 
leading hadrons $P_{1}$ and $P_{2}\left(P_{1}+P_{2}=P_{h}\right)$ are detected in the same jet, that acts as a spectator [6,7]. In this case, collinear factorization holds and the cross section would not be suppressed by Sudakov form factors; moreover, FSI are more easily modelled inside the pair. If the two hadrons are unpolarized, four FF appear at leading twist: $D_{1}, G_{1}^{\perp}, H_{1}^{\perp}, H_{1}^{\Varangle}$ [7]. They depend on the light-cone quark momentum fractions $z_{1}, z_{2}$ delivered to the hadrons $\left(z=\left(P_{1}+P_{2}\right)^{-} / k^{-}=z_{1}+z_{2}\right)$, on the transverse relative momentum $\vec{R}_{\perp}^{2}$ (with $R=\left(P_{1}-P_{2}\right) / 2$ ), on $\vec{k}_{\perp}^{2}$ and $\vec{k}_{\perp} \cdot \vec{R}_{\perp}[0]$. Each FF is also related to a specific spin state of the fragmenting quark: $H_{1}^{\perp}$ is the analogue of the Collins effect; on the contrary, $H_{1}^{\Varangle}$ represents a genuine new effect relating the transverse polarization of the fragmenting quark $\left(\vec{S}_{\perp}\right)$ to the transverse relative dynamics of the detected pair $\left(\vec{R}_{\perp}\right)$, i.e. it is an "analyzing power" that transforms $\vec{S}_{\perp}$ into the relative orbital angular momentum of the pair. $G_{1}^{\perp}, H_{1}^{\perp}, H_{1}^{\Varangle}$ are T-odd and are nonvanishing only in the presence of residual FSI, at least between the two hadrons. Both $H_{1}^{\perp}, H_{1}^{\Varangle}$ are also chiral odd and can be identified as the chiral partner needed to access the transversity $h_{1}$ [7].

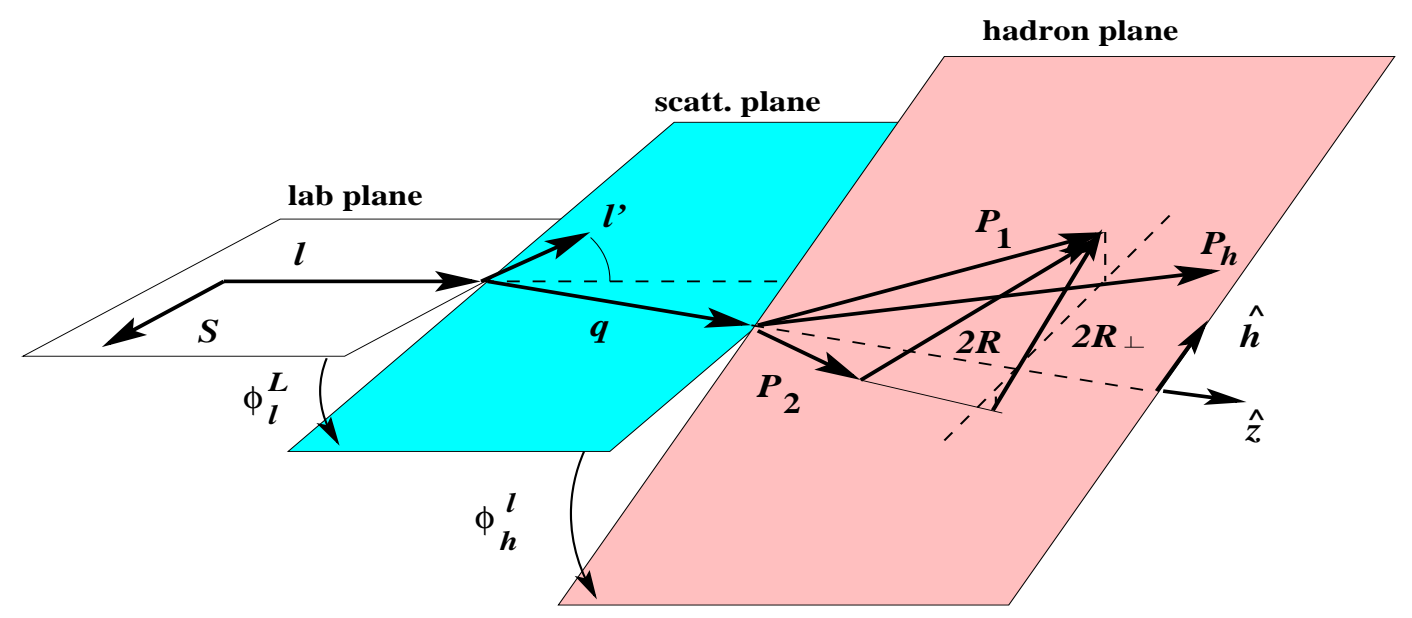

Figure 1. Kinematics for semi-inclusive DIS where two leading hadrons are detected (see text).

The cross section at leading twist for the process $e N \rightarrow e^{\prime} h_{1} h_{2} X$ has been worked out in detail in Ref. [7]. Here, we will reconsider the case for an unpolarized beam and a transversely polarized target. The lab frame can be defined by the plane containing the beam 3-momentum $\vec{l}$ and the target polarization $\vec{S}$ (see Fig. 迆. The scattering plane, which contains the scattered lepton 3 -momentum $\overrightarrow{l^{\prime}}$ and $\vec{l}$, is rotated by the azimuthal angle $\phi_{l}^{L}$. Finally, the socalled hadronic plane, which contains $\vec{q}=\vec{l}-\vec{l}^{\prime}$ and $\vec{P}_{h}$, is rotated by $\phi_{h}^{L}=\phi_{h}^{l}+\phi_{l}^{L}$. A further plane, which is just sketched in Fig. 11 for sake of simplicity, contains $\vec{P}_{1}, \vec{P}_{2}, \vec{R}$ and, consequently, $\vec{R}_{\perp}$; it is rotated by $\phi_{R}^{L}=\phi_{R}^{l}+\phi_{l}^{L}$ with respect to the lab. The nine-fold differential cross section depends on the energy fraction taken by the scattered lepton $\left(y=q^{0} /|\vec{l}|\right)$, on $\phi_{l}^{L}$, on the quark light-cone momentum fraction $x=p^{+} / P^{+}$of the target momentum $P$, on $z, \xi=z_{1} / z, \vec{R}_{\perp}$ and $\vec{P}_{h \perp}$. Since $R_{\perp}^{2}=\xi(\xi-1) P_{h}^{2}-(1-\xi) P_{1}^{2}-\xi P_{2}^{2}[7]$, the cross section can be more conveniently considered differential with respect to the pair invariant mass $P_{h}^{2}=M_{h}^{2}$ and $\phi_{R}^{L}$. By integrating over the "internal" dynamics (i.e. on $\xi, \vec{k}_{\perp}, \vec{P}_{h \perp}$ ) and by properly folding the 


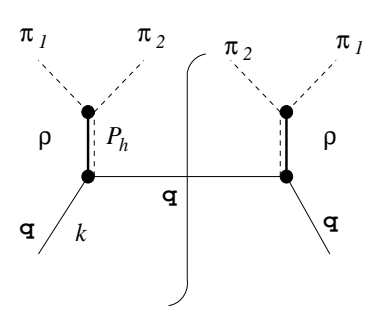

a

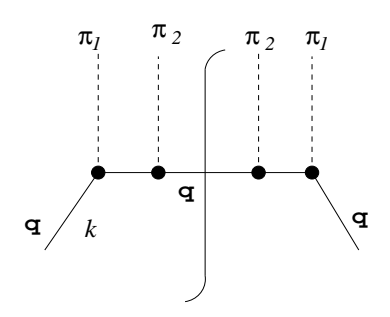

$\mathrm{b}$

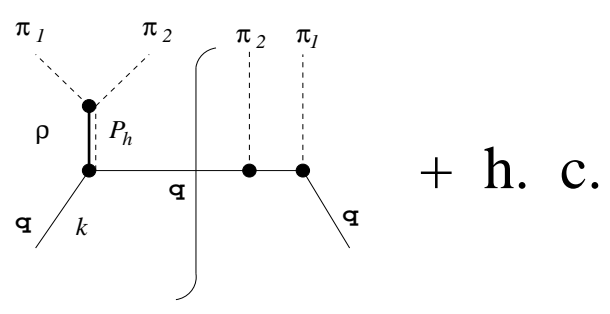

$\mathrm{C}$

Figure 2. The diagrams included for semi-inclusive detection of two pions with invariant mass within the $\rho$ resonance width and in the spectator approximation.

cross section over the experimental set of beam and hadron-pair azimuthal positions $\phi_{l}^{L}$ and $\phi_{R}^{L}$, it is possible to come to the factorized expression

$$
\begin{aligned}
\frac{\left\langle d \sigma_{O T}\right\rangle}{d y d x d z d M_{h}^{2}} \equiv & \int_{0}^{2 \pi} d \phi_{l}^{L} d \phi_{R}^{L} \sin \left(\phi_{R}^{L}-2 \phi_{l}^{L}\right) \int d \vec{P}_{h \perp} d \xi \frac{d \sigma_{O T}}{d y d \phi_{l}^{L} d x d z d \xi d M_{h}^{2} d \phi_{R}^{L} d \vec{P}_{h \perp}} \\
= & \frac{4 \pi \alpha_{e m}^{2} s}{(2 \pi)^{3} Q^{4}} \sum_{a} e_{a}^{2} x \frac{(1-y)\left|\vec{S}_{\perp}\right|}{2\left(M_{1}+M_{2}\right)} \int d \vec{p}_{\perp} h_{1}^{a}\left(x, \vec{p}_{\perp}^{2}\right) \\
& \quad \times \int d \xi\left|\vec{R}_{\perp}\right| \int_{0}^{2 \pi} d \phi_{R}^{L} \int d \vec{k}_{\perp} H_{1}^{\Varangle a}\left(z, \xi, M_{h}^{2}, \vec{k}_{\perp}^{2}, \vec{k}_{\perp} \cdot \vec{R}_{\perp}\right) \\
= & \frac{\alpha_{e m}^{2} s}{4 \pi^{2} Q^{4}} \frac{(1-y)\left|\vec{S}_{\perp}\right|}{M_{1}+M_{2}} \sum_{a} e_{a}^{2} x h_{1}^{a}(x) H_{1}^{\Varangle a}\left(z, M_{h}^{2}\right)
\end{aligned}
$$

where $\alpha_{e m}$ is the electromagnetic fine structure constant, $s=Q^{2} / x y=-q^{2} / x y$ is the total energy in the center-of-mass frame, $M_{1}, M_{2}$ are the masses of the two observed hadrons, and a sum over the flavor $a$ of each quark and antiquark with charge $e_{a}$ is performed (see also Ref. [8]). In general, the integrated function $H_{1}^{\Varangle}$ will depend on both $z$ and $M_{h}^{2}$, contrary to the assumption proposed in Ref. [6]. It is worth noting also that Eq. (1) suggests a simpler measurement with respect to the case where only one hadron is detected. In fact, here only the azimuthal position $\phi_{R}^{L}$ of the pair is required, while in order to measure the Collins effect both the azimuthal angle and the $\left|\vec{P}_{h \perp}\right|$ hadron momentum need to be known (see Ref. [4] and references therein).

Quantitative predictions for $H_{1}^{\Varangle a}$ in Eq. (II) can be produced by extending the spectator model of Ref. [9] to the case of the emission of a hadron pair. For the hadron pair being a proton and a pion, results have been published in Ref. [10], where FSI arise from the interference between the direct production and the Roper decay. Here, results are shown for the case of two pions with invariant mass in the range $\left[m_{\rho}-\Gamma_{\rho}, m_{\rho}+\Gamma_{\rho}\right]$, with $m_{\rho}=768$ $\mathrm{MeV}$ and $\Gamma_{\rho} \sim 250 \mathrm{MeV}$. The spectator state has the quantum numbers of an onshell quark with constituent mass $m_{q}=340 \mathrm{MeV}$. The quark decay at leading twist with the minimal number of vertices is represented by the set of diagrams shown in Fig. 2, where now T-odd FF arise from the contribution of diagram $2 \mathrm{c}$ through the interference between the direct production of the two $\pi$ and the $\rho$ decay. Explicit check has been made that the direct diagrams $2 \mathrm{a}$ and $2 \mathrm{~b}$ qualitatively reproduce the experimental strength in the $P$ and $S$-channels, respectively, which represent most of the total $\pi-\pi$ production strength. 
In Fig. 3, $\alpha_{e m}^{2} /\left[8 \pi^{2} m_{\pi}\right] \times H_{1}^{\Varangle}\left(z, M_{h}^{2}=m_{\rho}^{2}\right)$ of Eq. (1) is shown for the case $u \rightarrow \pi^{+} \pi^{-}$. It shows that a nonvanishing integrated interference FF survives allowing for the extraction of $h_{1}$ at leading twist, as suggested by Eq. (1).

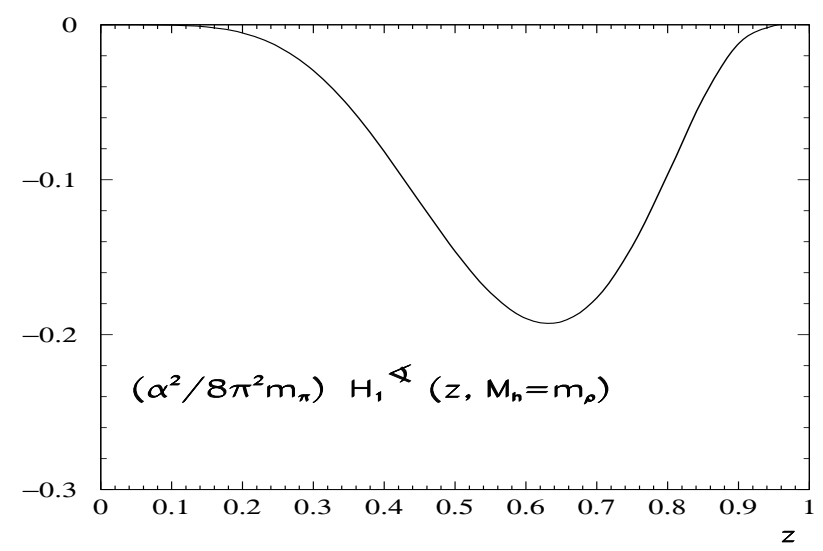

Figure 3. The fragmentation function $\alpha_{e m}^{2} /\left[8 \pi^{2} m_{\pi}\right] \times H_{1}^{\Varangle}\left(z, M_{h}^{2}=m_{\rho}^{2}\right)$ of Eq. (1) for the $u \rightarrow \pi^{+} \pi^{-}$case.

It is interesting to note that charge conservation for the diagrams of Fig. 2 implies that the same results are obtained for the processes $u \rightarrow \pi^{+} \pi^{-}$and $d \rightarrow \pi^{-} \pi^{+}$, which differ just by the interchange $\vec{R}_{\perp} \leftrightarrow-\vec{R}_{\perp}$. Therefore, the $\vec{k}_{\perp}$-integrated amplitudes for the $u$ and $d$ quarks leading to the same $\pi^{+} \pi^{-}$final state cancel each other. If only the valence quark content is considered for the proton $(p)$ and neutron $(n)$ targets, then the cross section at leading twist for the $e p \rightarrow e^{\prime} \pi^{+} \pi^{-} X$ process turns out the same as for the $e n \rightarrow e^{\prime} \pi^{-} \pi^{+} X$ one. Experimental tests of this conjecture could shed light both on the validiy of the spectator approximation and on the kinematical range where only the leading twist can be safely considered.

\section{ACKNOWLEDGMENTS}

This work has been done in collaboration with A. Bianconi (Univ. Brescia) and R. Jakob (Univ. Wuppertal). Enlighting discussions with S. Boffi (Univ. Pavia) and D. Boer (RIKEN-BNL) are greatfully acknowledged.

\section{REFERENCES}

1. A. de Rújula, J.M. Kaplan and E. de Rafael, Nucl. Phys. B 35 (1971) 365.

2. J.C. Collins and D.E. Soper, Nucl. Phys. B 194 (1982) 445.

3. J.C. Collins, Nucl. Phys. B 396 (1993) 161.

4. D. Boer, hep-ph/0102071.

5. A. Bacchetta, R. Kundu, A. Metz and P.J. Mulders, hep-ph/0102278.

6. R.L. Jaffe, X. Jin and J. Tang, Phys. Rev. Lett. 80 (1998) 1166.

7. A. Bianconi, S. Boffi, R. Jakob and M. Radici, Phys. Rev. D 62 (2000) 034008.

8. V. Barone, A. Drago and P.G. Ratcliffe, hep-ph/0104283.

9. R. Jakob, P.J. Mulders and J. Rodrigues, Nucl. Phys. A 626 (1997) 937.

10. A. Bianconi, S. Boffi, R. Jakob and M. Radici, Phys. Rev. D 62 (2000) 034009. 